\title{
Ventilated cavitating flow over a bluff body with special emphasis on the vortex-cavitation interaction
}

\author{
Zheng Yanan ${ }^{\mathrm{a}}$, Wang Zhiying ${ }^{\mathrm{b}, *}$, Wang Guoyu ${ }^{\mathrm{a}}$ \\ ${ }^{a}$ School of Mechanical Engineering, Beijing Institute of Technology, Beijing, 100081, China \\ ${ }^{\mathrm{b}}$ Key Laboratory for Mechanics in Fluid Solid Coupling Systems, Institute of Mechanics, Chinese Academy of Sciences, Beijing, 100190, China
}

\section{A R T I C L E I N F O}

\section{Keywords:}

Ventilated cavitating flow

Turbulent kinetic energy

Vortex identification

Vortex-cavitation interaction

\begin{abstract}
A B S T R A C T
The objective of this paper is to investigate the vortex-cavitation interaction in ventilated cavitating flow over a bluff body at Reynolds number $R e=6.7 \times 10^{4}$ by numerical method. The results present that in the active cavitation area, the turbulent kinetic energy mainly distributes in the leading edge of the cavity and the gasliquid interface of the shedding vortex. The intensity variation is closely related to the cavity morphology and the ventilated cavitation development. With the increase of the gas entrainment coefficient $Q_{v}$, the ventilated cavitation reduces the turbulence intensity. Three well-employed vortex identification methods, $\omega_{z}$ criterion, $Q$ criterion and the improved $\Omega$ method, are applied to identify the vortex structures. The comparison shows that the improved $\Omega$ method can well capture the vortices of different intensity in the cavitating flow field without using the threshold value, especially for the formation of the vortices. As for the ventilated cavitation vortex dynamics, strong rotation appears with the shear layer rolling up and relatively strong shear occurs with the entrainment of vortices during the vortex formation. With the increase of $Q_{v}$, both the rotation and shear in the active cavitation area tend to become weaken owing to the reduction of turbulent intensity.
\end{abstract}

\section{Introduction}

Ventilated cavitation can be obtained by injecting gas into the lowpressure regions in the flow, which can effectively reduce either the risk of erosion or the energy losses in variety of applications (Amromin et al., 2011; Hrubes, 2001; Michel, 1984). It attracts a lot of interest due to its practical importance in engineering and scientific relevance in fluid mechanics (Arndt et al., 2009; Bin et al., 2010; Ceccio, 2010; Skidmore et al., 2016). However, when the gas entrainment coefficient is not large enough to preserve the stable ventilated supercavity, the ventilated cavity is often accompanied with an unsteady behavior, including the formation, evolution and shedding of local vortex structures (Belahadji et al., 1995; Harwood et al., 2016; Kinzel et al., 2017; Wang et al, 2017, 2018), and the interactions between cavitation and vortex structures. In order to better understand and control the ventilated cavitating flow in the turbulent wake, it is necessary to investigate the ventilated cavitation vortex dynamics.

As ventilated cavitating flow is a complex turbulence flow with multiphase and multiscale vortex, various experiments and numerical methods have been conducted to investigate the unsteady behavior and the vortex structures in the wake (Chen et al., 2019; Cheng et al., 2018;
Huang et al., 2014; Long et al., 2018; Wu et al., 2018). Barbaca et al. (2019) carried out experiments in a high-speed water tunnel to investigate the topology and unsteady behavior of ventilated and natural cavity flows over a two-dimensional (2-D) wall-mounted fence. They found that both the size and the shed structures increase with the increase of free-stream velocity for ventilated cavitating flow. Brandao et al. (2020) studied the effect of non-condensable gas in the shedding characteristics of the cavitating flow over a circular cylinder. The results indicated that the injection of non-condensable gas suppresses the low frequency vortex shedding. Pendar and Roohi (2018) shed light on the cavitation characteristics around a sphere and indicated that the occurrence of cavitation suppresses turbulence and delays the breakdown of the vortices. Ausoni et al. (2007) mainly investigated the effects of cavitation and fluid-structure interaction on the vortex generation mechanism. They found that the vortex shedding frequency increased up to $15 \%$ due to the increase of the vorticity caused by cavitation. Ghahramani et al. (2020) combined experimental and numerical methods to investigate the cavitating flow around a bluff body and captured kinds of vortices for different cavitating flow patterns. Cavitation inception appears along with the streamwise and spanwise vortices. However, with the development of cavitation, except for streamwise and spanwise

\footnotetext{
* Corresponding author.

E-mail address: wangzhiying@imech.ac.cn (W. Zhiying).
} 
vortices, there also exists the transverse vortices and horseshoe vortices in the wake.

In order to identify and visualize the vortex structures in the cavitating turbulence flow, the definition of a vortex becomes a key issue. For a long time, a large number of scholars used vorticity to track vortex, and even directly regarded the region with large vorticity distribution as vortex (Babiano et al., 1987; Green, 2012; Wu et al., 2007). However, Robinson (1990) emphasized that in the turbulent boundary layer, the relationship between the region with large vorticity and the actual vortex structure may be very weak, especially in the near wall region. It means that a vortex cannot be fully described by vorticity. Vorticity can be decomposed into rotational and non-rotational part, which represents the rotation and the shear, respectively (Tian et al., 2018). So various other vortex identification methods have been proposed to recognize the vortex, including the $\Delta$ criteria (Perry and Chong, 1987), the $\lambda_{c i}$ method extended on the basis of $\Delta$ (Zhou et al., 1999), the Q method (Hunt et al., 1988), and the $\lambda_{2}$ criteria (Jeong and Hussain, 1995), etc. Perry and Chong (1987) proposed the $\Delta$ method in 1987, which defines the vortex as the region where the eigenvalues of the velocity gradient tensor are complex. Zhou et al. (1999) further developed the $\Delta$ method, namely the $\lambda_{c i}$ method, which suggested that the vortex exists when there are two complex eigenvalues in the velocity gradient tensor. Hunt et al. (1988) introduced the $Q$ method around the same time, arguing that there is a vortex structure in the region with positive second invariant $Q$ of the velocity gradient tensor. Although a variety of methods have been proposed to characterize the vortex structure based on Euler velocity field, these methods are sensitive to the threshold value. A larger threshold value will smear the weak vortex structure in the flow field, while a smaller threshold value will blur the vortex structure in the flow field. The problem is that there is no such rigorous criterion to determinate the threshold value, only by experience. In recent years, Liu et al. (2016) proposed an improved $\Omega$ method based on the $Q$ method to study the vortex dynamics and turbulence. The $\Omega$ method applies a determinative value to capture the vortex with a normalized value ranging from 0 to 1 that can simultaneously capture both the strong and weak vortices (Liu et al., 2019), which improves the identification of small vortex structure to a certain extent. Owing to its apparent superiority, the $\Omega$ method has been intensively employed by many researchers. Zhang et al. (2018) analyzed the vortex structures in the reversible pump turbine under typical operational modes using the $\Omega$ method. They found that the $\Omega$ method could capture both the weak and strong vortices in the whole passage of the reversible pump turbine, even if the vortex structure in the reversible pump turbine is very complex. Wang et al. (2019) used different vortex identification methods to investigate the cavitating flow structures around 2D Clark-Y hydrofoil and they also got the same conclusion for the advantage of the $\Omega$ method.

In the present work, the ventilated cavitating flow over a bluff body is investigated to reveal the physical interaction between the cavitation and vortex dynamics. The objectives of the present paper are to 1) investigate the unsteady characteristics of the ventilated cavitating flow, specifically in the cavity morphology and the evolution of cavitation vortex with different gas entrainment coefficients, 2) address the application of different vortex identification methods including $\omega_{z}$ criterion, $Q$ criteria, and $\Omega$ method in the ventilated cavitating flows, 3 ) elucidate the ventilated cavitation vortex dynamics, with special emphasis on the turbulent vortex-cavitation interactions.

\section{Numerical method}

\subsection{Numerical model}

The homogeneous model is adopted, which assumes that the gasliquid two-phase mixture is a homogeneous medium without any relative velocity between the two phases. Applying a Favre-filtering operation to the $N-S$ equations, the governing equations for the large eddy simulation is expressed as (Nicoud and Ducros, 1999): $\frac{\partial \rho_{m}}{\partial t}+\frac{\partial\left(\rho_{m} \bar{u}_{j}\right)}{\partial x_{j}}=0$

$\frac{\partial\left(\rho_{m} \bar{u}_{i}\right)}{\partial t}+\frac{\partial\left(\rho_{m} \bar{u}_{i} \bar{u}_{j}\right)}{\partial x_{j}}=\rho_{m} f-\frac{\partial p}{\partial x_{i}}+\frac{\partial}{\partial x_{j}}\left(\mu \frac{\partial \bar{u}_{i}}{\partial x_{j}}\right)-\frac{\partial \tau_{i j}}{\partial x_{j}}$

Here, the subscripts $i$ and $j$ represent the coordinate directions, $\bar{u}_{i}$ is the filtered velocity. The mixture density $\rho_{m}$ is defined as $\rho_{m}=\rho_{l} \alpha_{l}+$ $\rho_{g} \alpha_{g}$, and the mixture dynamic viscosity $\mu_{m}$ is defined as $\mu_{m}=\mu_{l} \alpha_{l}+$ $\mu_{g} \alpha_{g}$, where $\rho_{l}$ is the liquid density, $\rho_{g}$ is the gas density, $\mu_{l}$ is the liquid dynamic viscosity, $\mu_{g}$ is the gas dynamic viscosity, $\alpha_{l}$ is the liquid fraction, $\alpha_{g}$ is the gas fraction, and $\alpha_{l}=1-\alpha_{g} . \tau_{i j}$ is the sub-grid scale (SGS) stress defined as: $\tau_{i j}=\rho \overline{u_{i} u_{j}}-\bar{u}_{i} \bar{u}_{j}$. In the eddy viscosity approach, the subgrid-scale $\tau_{i j}$ is related to the large-scale rate tensor $\bar{S}_{i j}$ as followed:

$\tau_{i j}-\frac{1}{3} \tau_{k k} \delta_{i j}=-2 \mu_{t} \bar{S}_{i j}$

$\bar{S}_{i j}=\frac{1}{2}\left(\frac{\partial \overline{u_{i}}}{\partial x_{j}}+\frac{\partial \overline{u_{j}}}{\partial x_{i}}\right)$

Establishing a relationship between $\mu_{t}$ and $S_{i j}$ with the Wall-Adapting Local Eddy-Viscosity (WALE) model (Nicoud and Ducros, 1999):

$\mu_{t}=\rho_{m} L_{s}^{2} \frac{\left(S_{i j}^{d} S_{i j}^{d}\right)^{3 / 2}}{\left(\bar{S}_{i j} \bar{S}_{i j}\right)^{5 / 2}+\left(S_{i j}^{d} S_{i j}^{d}\right)^{5 / 4}}$

$S_{i j}^{d}=\frac{1}{2}\left(\bar{g}_{i j}^{2}+\bar{g}_{i j}^{2}\right)-\frac{1}{3} \delta_{i j} \bar{g}_{k k}^{2}, \bar{g}_{i j}^{2}=\bar{g}_{i k} \bar{g}_{k j}, \bar{g}_{i j}=\frac{\partial \bar{u}_{i}}{\partial x_{j}}$

$L_{s}=\min \left(k d, C_{s} V^{1 / 3}\right)$

where, $S_{i j}^{d}$ denotes the traceless symmetric part of the square of the velocity gradient tensor, $L_{s}$ is the mixed length of the sub-grid scale, $k$ is von Karman's constant, $C_{s}$ is a constant calculated as $0.35, d$ is the minimum distance from the grid point to the wall, $V$ is the volume of the computational cell.

\subsection{Numerical setup}

A schematic of the calculation mesh and the illustration of boundary conditions imposed for the test body are shown in Fig. 1. The diameter of the bluff body is $D=0.02 \mathrm{~m}$, the bluff body is $9.5 D$ in height, $35 D$ in width, and the spanwise length is $0.2 D$. The downstream-side surface is half-cylinder, on which a row of orifices with $d=0.1 D$ are distributed uniformity. A no-slip boundary condition is imposed on the test body surface. All cases are simulated based on the experiments of Wang et al. (2018). The Reynolds number $R e$ and the gas entrainment coefficient $Q_{v}$ are defined as:

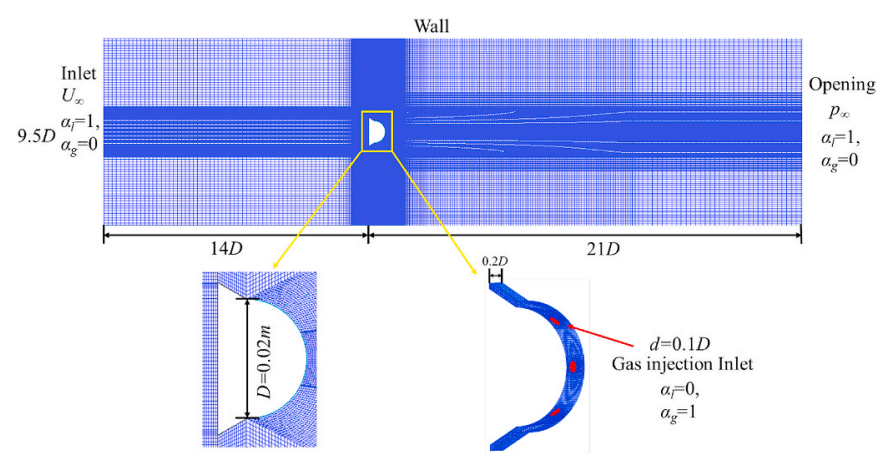

Fig. 1. Calculation mesh and boundary conditions. 


$$
\operatorname{Re}=U_{\infty} D / \nu
$$

$Q_{v}=Q / U_{\infty} D^{2}$

where, $U_{\infty}$ is the free stream velocity, $\nu$ is the viscosity coefficient of water, $Q$ is the volumetric flow rate of the injected gas and $D$ is the diameter of the body.

\section{Typical vortex identification methods}

\section{1. $\omega$ criterion}

The vorticity is defined as the curl of the fluid velocity vector (Ghahramani et al., 2020), which is regarded as one of the important physical quantities describing the vortex motion.

$\omega_{z}=\frac{1}{2}\left(\frac{\partial v}{\partial x}-\frac{\partial u}{\partial y}\right)$

$\left|\omega_{z}\right|>\omega_{\text {thresh }}$

The vorticity magnitude $\left|\omega_{z}\right|$ is twice as much as the average angular velocity of the fluid element and can reflect the average rotation of the fluid to some extent. The $\omega$ criterion asserts that a vortex exists when the vorticity magnitude is larger than the threshold, as shown in Eq. (11). But this method cannot always capture the vortex structures very well. For example, in the laminar boundary layer, though the vorticity magnitude is large enough due to the high-velocity gradient, there is not any vortex rotation. Besides, the threshold is arbitrary, without any rigorous criterion.

\subsection{Q criterion}

Proposed by Hunt et al. (1988), the $Q$ method represents the balance between shear strain rate and vorticity. For the incompressible flow, the $Q$ criterion can be defined as:

$Q=\frac{1}{2}\left(\|B\|_{F}^{2}-\|A\|_{F}^{2}\right)$

Where $\|\cdot\|_{F}$ is the Frobenius norm, $A$ and $B$ are respectively the symmetric part and antisymmetric part of the velocity gradient tensor, $\nabla \boldsymbol{V}$.

$\nabla V=\frac{1}{2}\left(\nabla V+\nabla V^{T}\right)+\frac{1}{2}\left(\nabla V-\nabla V^{T}\right)=A+B$

$A=\frac{1}{2}\left(\nabla V+\nabla V^{T}\right)=\left[\begin{array}{ccc}\frac{\partial u}{\partial x} & \frac{1}{2}\left(\frac{\partial u}{\partial y}+\frac{\partial v}{\partial x}\right) & \frac{1}{2}\left(\frac{\partial u}{\partial z}+\frac{\partial w}{\partial x}\right) \\ \frac{1}{2}\left(\frac{\partial v}{\partial x}+\frac{\partial u}{\partial y}\right) & \frac{\partial v}{\partial y} & \frac{1}{2}\left(\frac{\partial v}{\partial z}+\frac{\partial w}{\partial x}\right) \\ \frac{1}{2}\left(\frac{\partial w}{\partial x}+\frac{\partial u}{\partial z}\right) & \frac{1}{2}\left(\frac{\partial w}{\partial y}+\frac{\partial v}{\partial z}\right) & \frac{\partial w}{\partial z}\end{array}\right]$

$B=\frac{1}{2}\left(\nabla V-\nabla V^{T}\right)=\left[\begin{array}{ccc}0 & \frac{1}{2}\left(\frac{\partial u}{\partial y}-\frac{\partial v}{\partial x}\right) & \frac{1}{2}\left(\frac{\partial u}{\partial z}-\frac{\partial w}{\partial x}\right) \\ \frac{1}{2}\left(\frac{\partial v}{\partial x}-\frac{\partial u}{\partial y}\right) & 0 & \frac{1}{2}\left(\frac{\partial v}{\partial z}-\frac{\partial w}{\partial x}\right) \\ \frac{1}{2}\left(\frac{\partial w}{\partial x}-\frac{\partial u}{\partial z}\right) & \frac{1}{2}\left(\frac{\partial w}{\partial y}-\frac{\partial v}{\partial z}\right) & 0\end{array}\right]$

It is supposed to be a vortex when it is positive, $Q>0$. The $Q$ criterion is mostly applied to the identification of the vortex structure in the incompressible flow, while it is also sensitive to the selection of the threshold.

\section{3. $\Omega$ method}

The vorticity can represent the rotation of the rigid body but not the rotation of the fluid, for the fluid movement inevitably contains shear and deformation. In recent years, a new vortex identification method proposed by Liu et al. (2019), the $\Omega$ method, believes that the specific gravity of fluid deformation is also not negligible. The $\Omega$ method represents the vortex as the area where the local vorticity overcomes the deformation of the local fluid, that is, the ratio of the square of the vorticity to the sum of the squares of the vorticity and the deformation, as shown in Eq. (16):

$\Omega=\frac{b}{a+b}$

Where, $a, b$ are the Frobenius norms of $A$ and $B$, respectively, which are positive numbers obviously.

$a=\operatorname{trace}\left(A^{T} A\right)=\sum_{i=1}^{3} \sum_{j=1}^{3} A_{i, j}^{2}$

$b=\operatorname{trace}\left(B^{T} B\right)=\sum_{i=1}^{3} \sum_{j=1}^{3} B_{i, j}^{2}$

To improve the robustness and accuracy, a new $\Omega$ method is proposed to be:

$\Omega=\frac{b}{a+b+\varepsilon}$

Where, $\varepsilon$ is a small positive number using to avoid the division by zero (2018), $\varepsilon=10^{-6 *}(b-a)_{\max }$ is used in this paper (Dong et al., 2018). The new $\Omega$ method can effectively avoid the influence when $a$ and $b$ is small enough to zero, in which the value of $\Omega$ may be big enough to cause noise or clouds in the vortex structure. Unlike other vortex identification methods described above, $\Omega$ is a normalized fraction of $0-1, \Omega=$ 0 means the pure shear existing in the flow field, while $\Omega=1$ means the pure rigid body rotation. The $\Omega$ method has no threshold limitation, with the value greater than 0.5 , it can well capture the vortex structure in the flow field. In addition, the method is applicable to both compressible and incompressible flows.

\section{Results and discussion}

Fig. 2 shows the typical cavity patterns with different Reynolds number $R e$ and gas entrainment coefficient $Q_{v}$ obtained from the experimental data (Wang et al., 2018). From the experimental results, the cavity topology can be classified into two types, namely cyclic states and relatively stable states. The specific definitions of cavity patterns in the map are given in Wang et al. (2018), and four typical cavity patterns are shown in the map. For the cyclic state, the cavity length is relatively

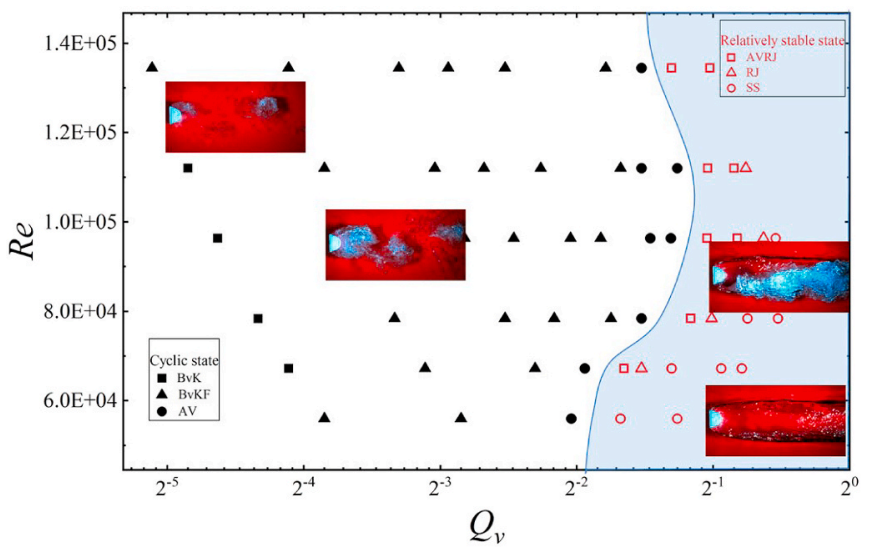

Fig. 2. Map of different flow structures for the ventilated cavitation. 
short and cavitation vortices alternately shed into the wake, forming cavitation vortex street. During the cycle, a strong vortex-cavitation interaction occurs. For the relatively stable state, observed for high values of $Q_{v}$, the cavity length becomes sufficiently large for the development of the re-entrant jet. The re-entrant jet plays an important role in the vortex shedding. With the gas entrainment coefficient $Q_{\nu}$ increases, the supercavity is gradually formed.

\subsection{Time evolution of ventilated cavity structures at typical regimes}

The cyclic ventilated cavitating flow is accompanied by the formation, development, and shedding of large-scale vortices. Fig. 3 presents the cavitation morphology and cavity structures during one cycle at $Q_{v}$ $=0.0866$ for $R e=6.7 \times 10^{4}$. The middle side frames demonstrate the iso-surfaces of the volume fractions. The grey iso-surface represents the gas volume fraction with $F=0.05$ and the blue iso-surface represents the gas volume fraction with $F=0.95$. The images in the right illustrate contours of turbulent kinetic energy (TKE). They are in good agreement with the observation in the experiments (Wang et al., 2018). At $t_{1}$, the gas is injected into the wake, forming the upper vortex. The upper shear layer entrains fluid from the adjacent flow, and the upper vortex prepares for shedding from the upper part of the bluff body. From the distribution of turbulent kinetic energy, it could be observed that, in the active cavitation area, the high level of turbulent kinetic energy mainly concentrates on the leading edge of the cavity (I) and the gas-liquid interface region of shedding vortices (II), while the turbulent intensity is relatively weak inside the cavity (IV). For the interstitial area (III), distribution of turbulent kinetic energy is large owing to the entrainment between the upper vortex and the lower vortex. At $t_{2}$, the upper vortex is cut off and it starts to shed into the wake. The lower shear layer begins to develop. The turbulent kinetic energy in the leading edge of the upper vortex decreases, while increases in the leading edge of the lower part. In the gas-liquid interface region, the turbulent kinetic energy increases. Then at $t_{3}$, the shedding upper vortex moves downstream with rotation, and the lower vortex begins to form with the air entrainment of the lower shear layer. At this instant, the turbulent kinetic energy in the leading edge of the lower cavity further increases, while increases to the maximum value in the gas-liquid interface region of the shedding vortices. At $t_{4}-t_{5}$, it can be found that the development process of the lower vortex is similar to that of the upper vortex, thereby a period of two opposite vortices alternately shedding is formed. The turbulent kinetic energy gradually decreases in the gas-liquid interface region as the lower vortex sheds.

In the case at $Q_{v}=0.231$ for $R e=6.7 \times 10^{4}$, the cavitating flow field is relatively stable, as show in Fig. 4. With the increase of gas entrainment coefficient $Q_{v}$, it is observed that an attached cavity forms and the
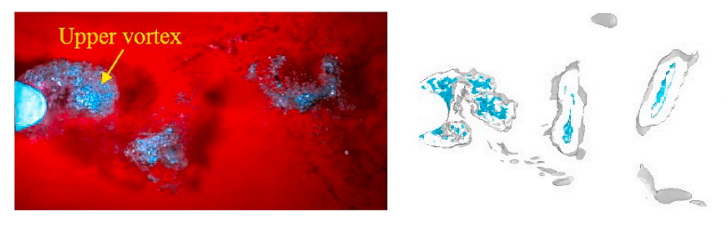

(a) $t_{1}=t_{0}$
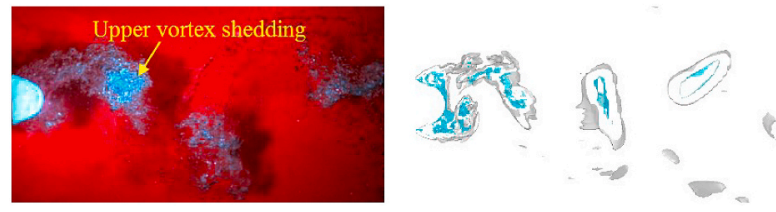

(b) $t_{2}=t_{0}+1 / 5 T$
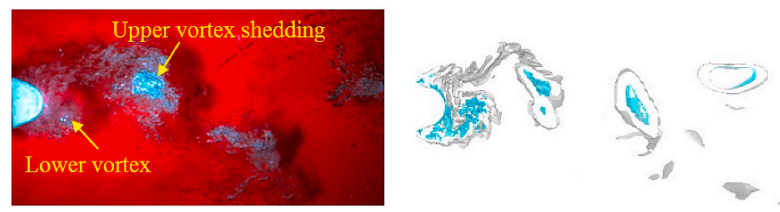

(c) $t_{3}=t_{0}+2 / 5 T$
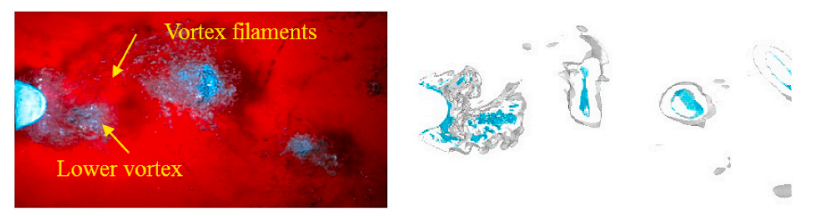

(d) $t_{4}=t_{0}+3 / 5 T$
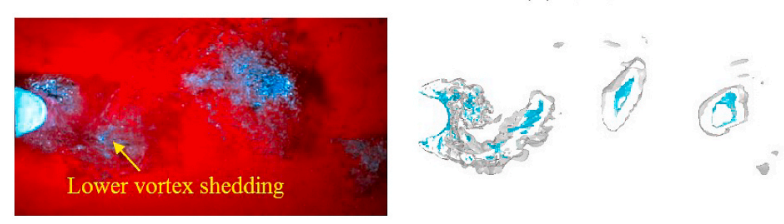

(e) $t_{5}=t_{0}+4 / 5 T$
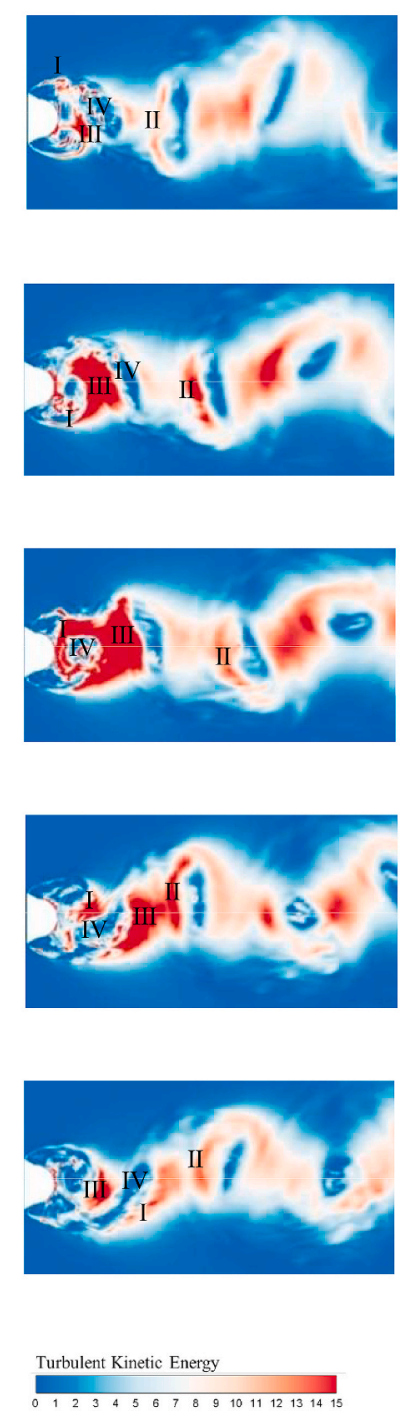

Fig. 3. The experimental images (left) (Wang et al., 2018), Iso-surfaces of the volume fractions (middle) and contours of turbulent kinetic energy (TKE) (right) at $Q_{v}$ $=0.0866$ for $R e=6.7 \times 10^{4}$. 
cavity length increases. The cloud cavity is shed from the rear of the cavity and becomes larger along the downstream direction. Compared with the case at $Q_{v}=0.0866$, as shown in Fig. 3, the distribution of turbulent kinetic energy is relatively smaller inside the cavity, that means an increase in $Q_{v}$ would promote the formation of a more stable cavity. Moreover, the reduction of turbulent kinetic energy implies that the shear layer weakly retains the fluid near the leading edge of the cavity in the active cavitation region, and the interaction between vortices and the fluid in the gas-liquid interface region becomes weak.

In order to give a quantitative comparison of turbulent kinetic energy distribution, Fig. 5 displays the variation of the turbulent kinetic energy along the flow direction at the section of $Y / D=0.5$ at $t_{1}$ and $t_{3}$ respectively, corresponding to the moment with the upper vortex forming and shedding. It indicates that the turbulent kinetic energy reaches the maximum value at the gas-liquid interface region of shedding vortices in the near wake and oscillates in the far wake due to the alternately shedding of cavitation vortices. From the turbulent kinetic energy distribution at the leading edge of the cavity in the active cavitation area, it can be found that the turbulent kinetic energy increases when the vortex forms (as shown in the $t_{3}$ ) and it gradually decreases when the vortex sheds. Besides, comparing the turbulent kinetic energy distribution for the cases with $Q_{v}=0.0866$ and $Q_{v}=0.231$, the turbulent kinetic energy becomes lower when $Q_{v}$ increases, which illustrates that

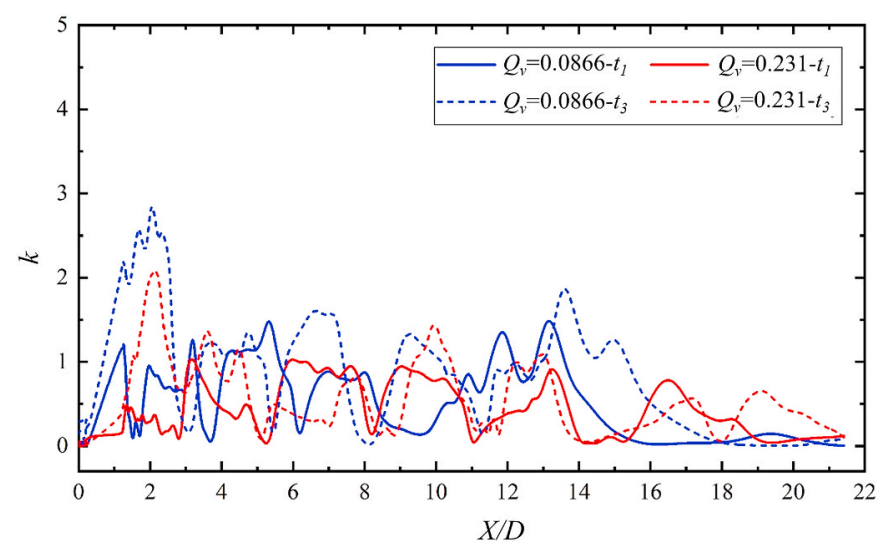

Fig. 5. Variation of Turbulent Kinetic Energy with cavitation development under different conditions (Section $Y / D=0.5$ ).
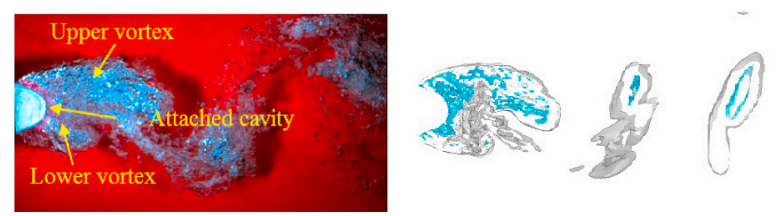

(a) $t_{1}=t_{0}$
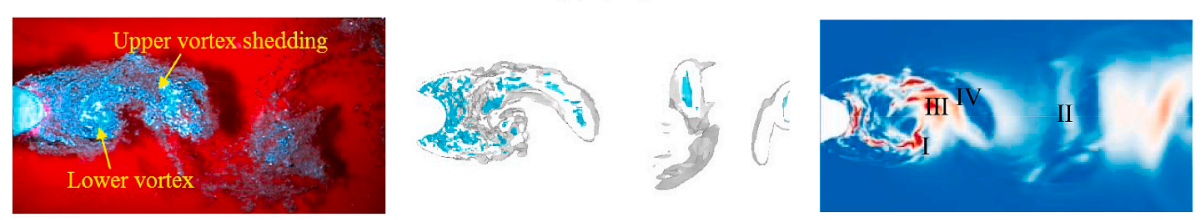

(b) $t_{2}=t_{0}+1 / 5 T$
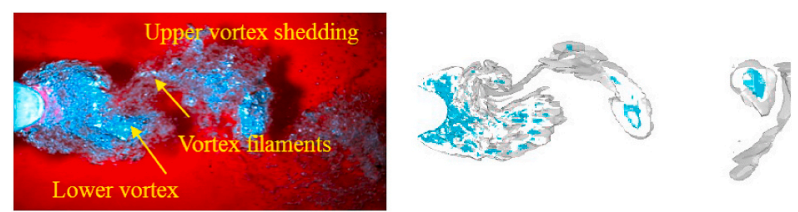

(c) $t_{3}=t_{0}+2 / 5 T$
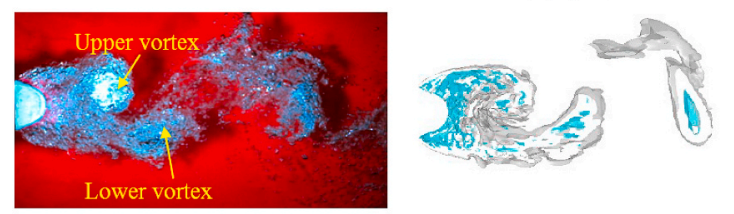

(d) $t_{4}=t_{0}+3 / 5 T$
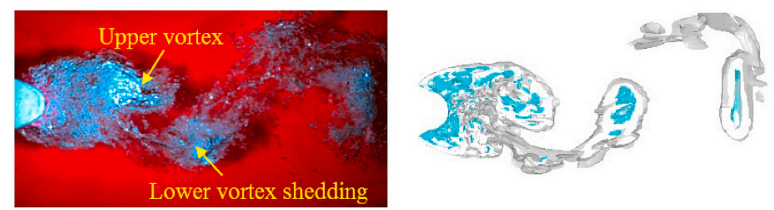

(e) $t_{5}=t_{0}+4 / 5 T$
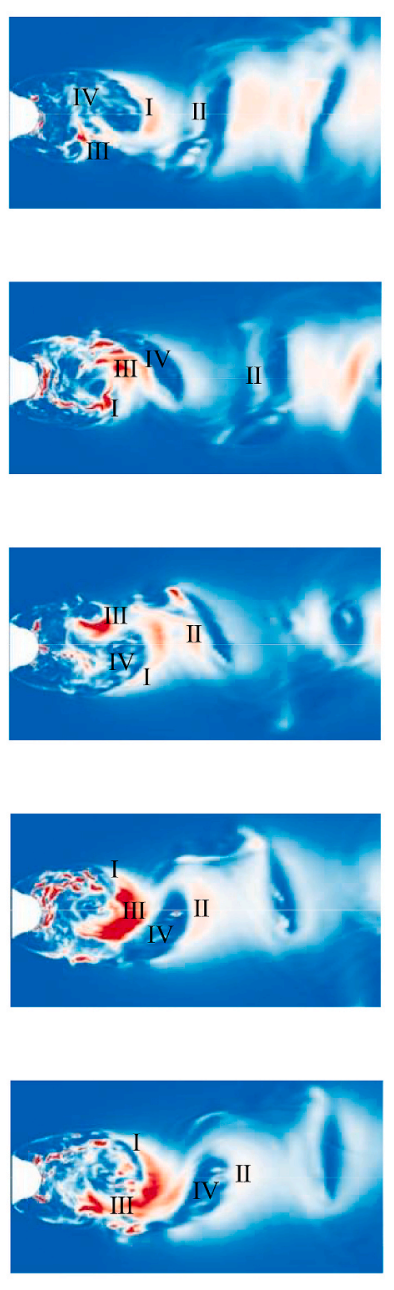

Turbulent Kinetic Energy

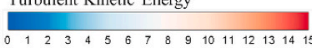

Fig. 4. The experimental images (left) (Wang et al., 2018), Isosurfaces of the volume fractions (middle) and contours of turbulent kinetic energy (TKE) (right) at $Q_{v}$ $=0.231$ and $R e=6.7 \times 10^{4}$. 
the turbulent intensity becomes weaker.

\subsection{Comparison of the flow structures with different identification} methods

Fig. 6 shows the vortex flow structures obtained by the above vortex identification methods under the typical cavitation pattern at $Q_{v}=0.231$ for $R e=6.7 \times 10^{4}$. The contour line represents the gas volume fraction with $F=0.01$. As shown in Fig. 6, when a proper threshold is chosen, the $\omega_{z}$ criterion can identify vortex structures of two opposite directions, as described above, the upper vortex and the lower vortex, while the $Q$ criterion can identify more vortex structures on the boundaries of the shedding vortex. It is noteworthy that the two methods only capture the strong vortex, skipping the weak vortex, resulting in some structures being smeared. For the $\Omega$ method, the identified vortex is universal. The comparisons between the $\omega_{z}, Q$ criterion and the $\Omega$ method are significantly demonstrated. It can be found that the $\Omega$ method could well capture vortices of different intensity simultaneously, especially for the weak vortex. In addition, the improved $\Omega$ method is applied here, successfully avoiding the pseudo-vortex structures or clouds in the flow field.
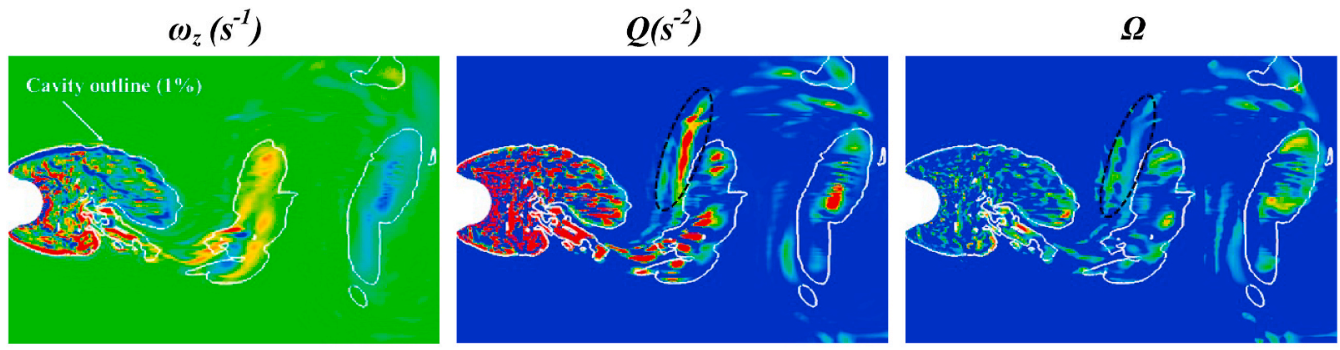

(a) $t_{1}=t_{0}$
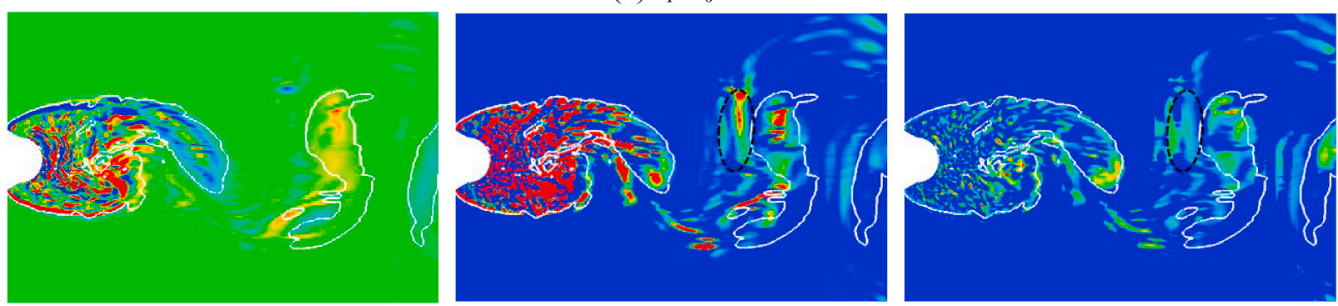

(b) $t_{2}=t_{0}+1 / 5 T$
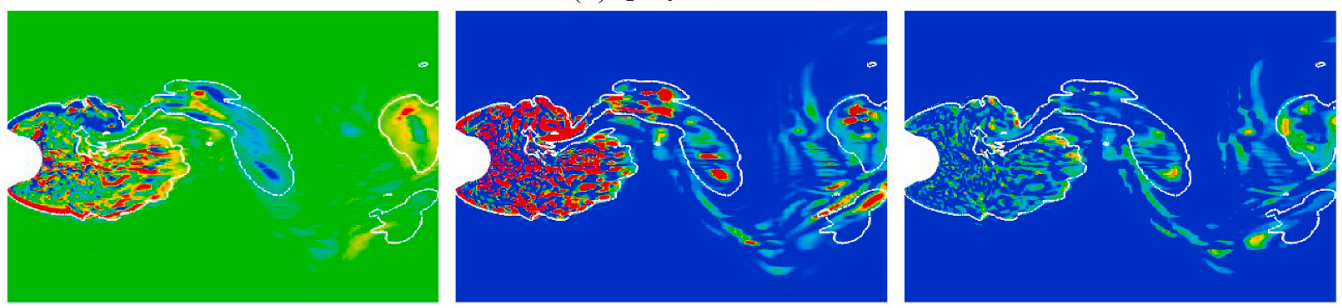

(c) $t_{3}=t_{0}+2 / 5 T$
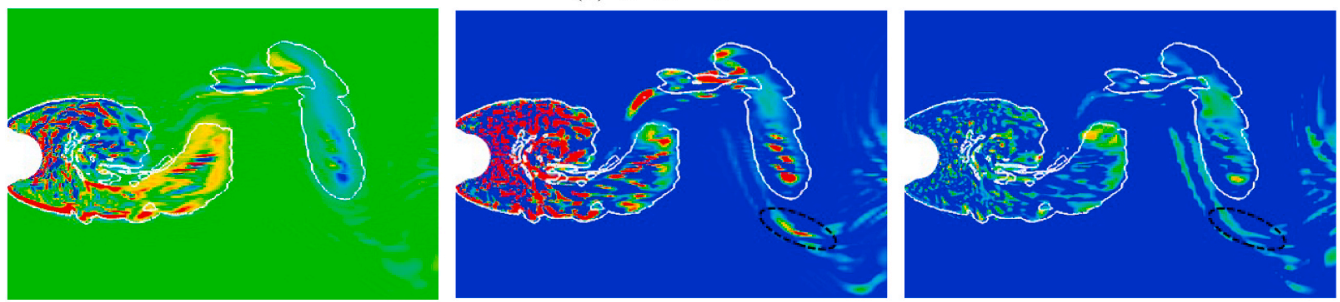

(d) $t_{4}=t_{0}+3 / 5 T$
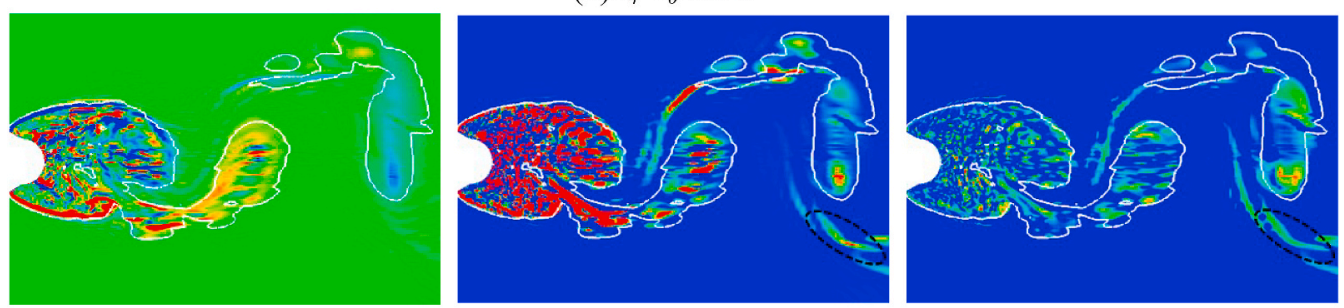

(e) $t_{5}=t_{0}+4 / 5 T$
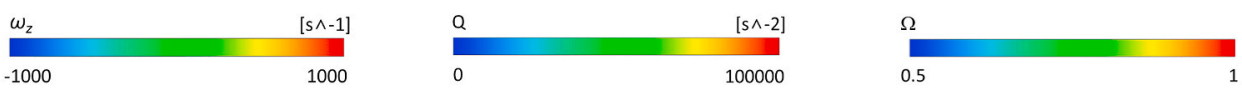

Fig. 6. Comparison of the vortex identification by the $\omega_{z}$ criterion (left), $Q$ criterion (middle) and $\Omega$ method (right) at $Q_{v}=0.231$ for $R e=6.7 \times 10^{4}$. 
4.3. Quantitive analysis of the ventilated cavity vortex flow structures with rotation and shear

Earlier work of Del Sordo and Brandenburg (2011) has demonstrated that vorticity production is actually quite ubiquitous once there is rotation, shear, or baroclinicity, while the effects of rotation and shear can contribute to the production of vorticity even in the absence of baroclinicity. According to Liu et al. (2019), vortex in fluid flow is always a mixture of vorticity and deformation. To elucidate the interrelationships between the effects of rotation and shear with the flow structures during the ventilated cavity vortex shedding, a research on the parameter $c$ defined as $c=10^{-6 *}(b-a)$ is conducted, where $a$ represents deformation while $b$ is related to the whole vorticity. In the turbulent shear flow, the shear strain leads to deformation, so $a$ could be believed as shear. Strictly speaking, vorticity could be considered as self-rotation for an infinitesimal particle rather than the global rotation, introduced by Liu et al. [28]. More specifically, vorticity can directly represent the rotation of a solid body, but not that of fluid. Nevertheless, in the turbulent flow, it is the vortical vorticity contributes to rotation. In general, $b$ could be believed as rotation here. In this section, two cyclic states including the Bénard-Kármán vortex street with vortex filaments (BvKF), the Aligned vortices (AV) and one relatively stable state, Stable Supercavity (SS) are discussed to deeply reveal the turbulent vortex-cavitation interactions.

Fig. 7 shows the gas volume fraction contour stacked with the defined parameter $c$ in the state of BvKF when $Q_{v}=0.0866$ and $R e=6.7$ $\times 10^{4}$, with the blue dotted line representing the position of $c=0$ and the corresponding scale size is shown below. When the gas entrainment coefficient is small, the shear and rotation near the orifices are very weak. In the vortex formation process, there is a strong rotation in the forming lower vortex as the lower shear layer rolls up and attains vorticity continuously from the front shear layer. Simultaneously, an extremely strong shear occurs in the forming lower vortex owing to the lower vortex entraining the upper vortex. In the vortex shedding process, the shedding vortices separated from the shear layer have an identical development process. In the wake, the relatively strong rotation would be gradually weakened as the vortex moves downstream, and the shear in the cavity gets weaken as well.

The comparison between the two cyclic states of $\mathrm{AV}$ at $Q_{v}=0.231$ and BvKF at $Q_{v}=0.0866$, shows that the effects of rotation and shear to the vortex flow structures are extremely similar. At $Q_{v}=0.231$, an attached cavity is formed by the gas ventilated, and the rotation near the orifices is strong when the ventilation rate increases, as shown in Fig. 8. It is relevant also, a relatively strong shear occurs at the vortex filament, owing to the lower vortex entraining the shedding upper vortex. In the vortex shedding process, compared to Fig. 7 , the rotation in the shedding vortex is significantly weakened.

At $Q_{v}=0.347$, the ventilated cavity is relatively stable, called Stable Supercavity (SS). As shown in Fig. 9, the shear near the orifices is extremely strong, compared with Figs. 7 and 8. In front of the supercavity, the shear is relatively strong due to the ventilated gas. In the transparent cavity, it is in a relatively stable state without any bubbles, only an extremely slight shear occurs in the distinct cavity interface of the upper and lower boundaries. At the tail of the supercavity, the gas moved along the cavity interface to the closure region of the cavity, is mixed with liquid, forming the small-scale bubbles. Thus, as Fig. 9 shows, the slight rotation occurs at the cavity boundary.

\section{Conclusions}

The characteristics of ventilated cavitating flow over a bluff body are investigated at Reynolds number $R e=6.7 \times 10^{4}$. The unsteady behavior, turbulent kinetic energy, vortex identification methods and vortex dynamics are described in detail. The main findings are as follows:

(1) The relationship between turbulent kinetic energy distribution and ventilated cavitation development in the cyclic ventilated cavitating flow are studied. The results show that in the active cavitation, the high level of turbulent kinetic energy mainly concentrates on the leading edge of the cavity and the gas-liquid interface region of shedding vortices. With the increase of gas entrainment coefficient $Q_{v}$, the turbulent intensity is effectively reduced. Consequently, the vortex-cavitation interaction becomes weaker and the flow fluid is more stable.

(2) The $\omega_{z}$ criterion, $Q$ criterion and the improved $\Omega$ methods, have been used to study the ventilated cavitating flow structures. It can be found that the $\Omega$ method can well capture vortices of different intensity without depending on the threshold value.

(3) The analysis of a defined parameter $c$ is discussed to deeply reveal the turbulent vortex-cavitation interactions. In the vortex formation process, strong rotation occurs with the rolling up of shear layer, and strong shear occurs with the entrainment of vortices. In the vortex shedding process, the rotation and shear gradually become weakened as the vortex moves downstream. For a supercavitation, it is relatively stable in the transparent cavity, only extremely sight shear occurs in the distinct cavity interfaces.

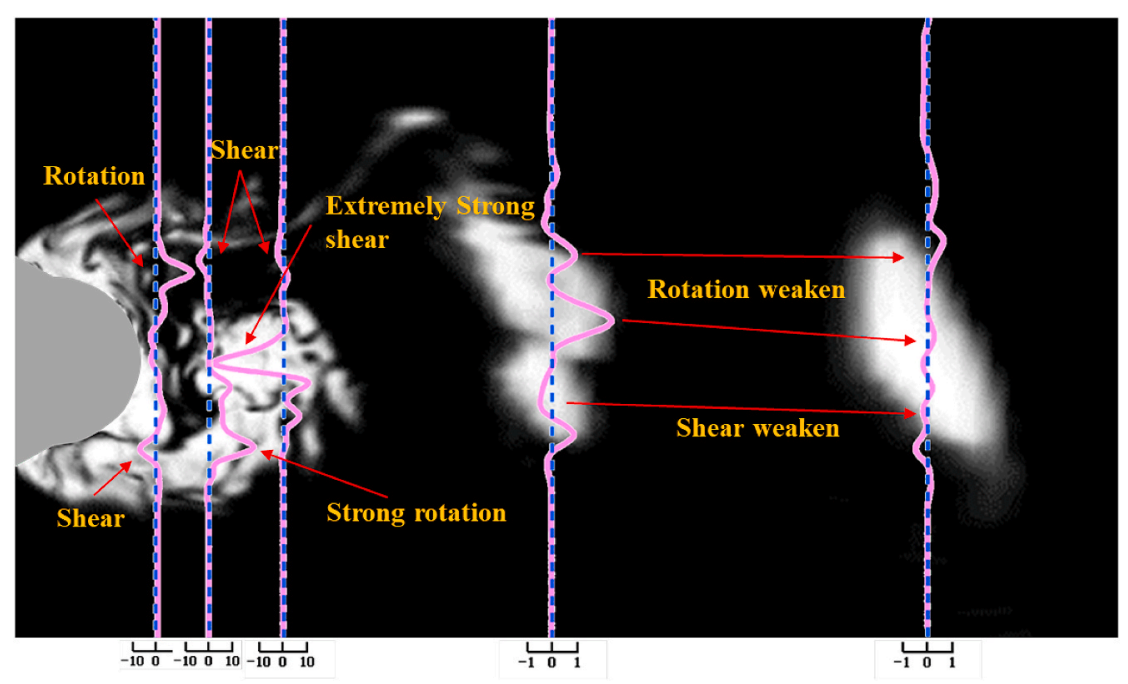

Fig. 7. Analysis of rotation and shear in the ventilated cavitation vortex dynamics at $Q_{v}=0.0866$. 


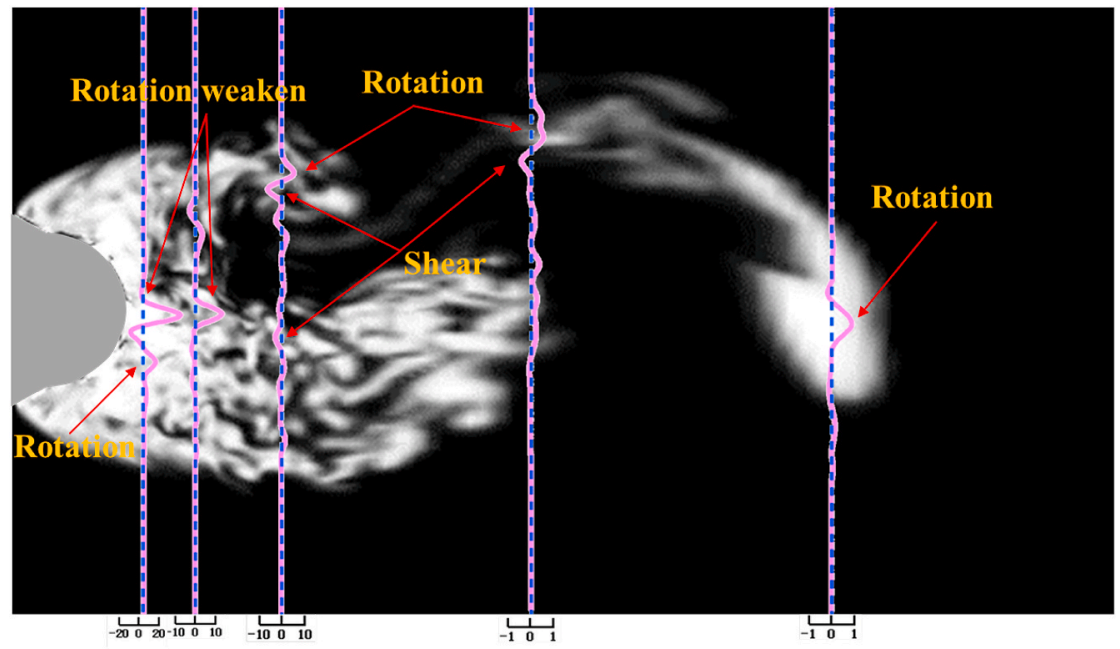

Fig. 8. Analysis of rotation and shear in the ventilated cavitation vortex dynamics at $Q_{v}=0.231$.

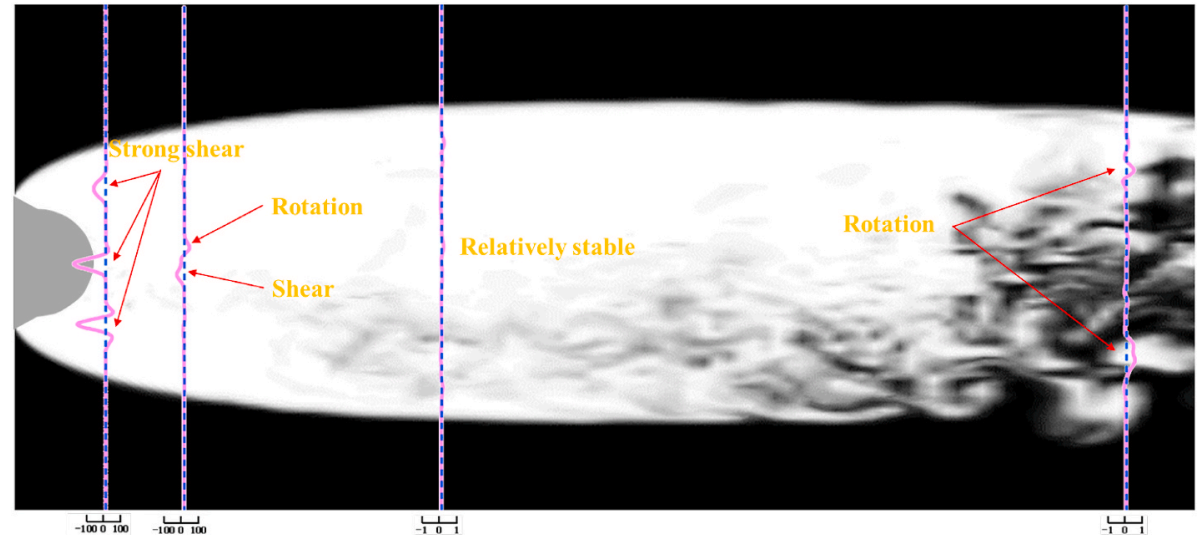

Fig. 9. Analysis of rotation and shear in the ventilated cavitation vortex dynamics at $Q_{v}=0.347$.

\section{CRediT authorship contribution statement}

Zheng Yanan: Investigation, Methodology, Formal analysis, Writing - original draft. Wang Zhiying: Conceptualization, Supervision, Writing - review \& editing, Resources. Wang Guoyu: Conceptualization, Supervision, Writing - review \& editing.

\section{Declaration of competing interest}

The authors declare that they have no known competing financial interests or personal relationships that could have appeared to influence the work reported in this paper.

\section{Acknowledgement}

The authors gratefully acknowledge support by the National Natural Science Foundation of China (Nos. 11902323 and 51839001).

\section{References}

Amromin, E., Karafiath, G., Metcalf, B., 2011. Ship drag reduction by air bottom ventilated cavitation in calm water and in waves. J. Ship Res. 55 (3), 196-207.

Arndt, R.E.A., Hambleton, W.T., Kawakami, E., Amromin, E.L., 2009. Creation and maintenance of cavities under horizontal surfaces in steady and gust flows. J. Fluid Eng. 131 (11).

Ausoni, P., Farhat, M., Escaler, X., Egusquiza, E., Avellan, F., 2007. Cavitation influence on von Kármán vortex shedding and induced hydrofoil vibrations. J. Fluid Eng. 129 (8), 966-973.
Babiano, A., Basdevant, C., Legras, B., Sadourny, R., 1987. Vorticity and passive-scalar dynamics in two-dimensional turbulence. J. Fluid Mech. 183, 379-397.

Barbaca, L., Pearce, B.W., Ganesh, H., Ceccio, S.L., Brandner, P.A., 2019. On the unsteady behaviour of cavity flow over a two-dimensional wall-mounted fence. J. Fluid Mech. 874, 483-525.

Belahadji, B., Franc, J.P., Michel, J.M., 1995. Cavitation in the rotational structures of a turbulent wake. J. Fluid Mech. 287, 383-403.

Bin, J.I., Luo, X.W., Peng, X.X., Zhang, Y., Wu, Y.L., Xu, H.Y., 2010. Numerical investigation of the ventilated cavitating flow around an under-water vehicle based on a three-component cavitation model. J. Hydrodyn. Ser. B 22 (6), 753-759.

Brandao, F.L., Bhatt, M., Mahesh, K., 2020. Numerical study of cavitation regimes in flow over a circular cylinder. J. Fluid Mech. 885.

Ceccio, S.L., 2010. Friction drag reduction of external flows with bubble and gas injection. Annu. Rev. Fluid Mech. 42, 183-203.

Chen, T., Chen, H., Liu, W., Huang, B., Wang, G., 2019. Unsteady characteristics of liquid nitrogen cavitating flows in different thermal cavitation mode. Appl. Therm. Eng. 156, 63-76.

Cheng, H.Y., Long, X.P., Ji, B., Liu, Q., Bai, X.R., 2018. 3-D Lagrangian-based investigations of the time-dependent cloud cavitating flows around a Clark-Y hydrofoil with special emphasis on shedding process analysis. J. Hydrodyn. 30 (1), $122-130$.

Del Sordo, F., Brandenburg, A., 2011. Vorticity production through rotation, shear, and baroclinicity. Astron. Astrophys. 528, A145.

Dong, X.R., Wang, Y.Q., Chen, X.P., Dong, Y., Zhang, Y.N., Liu, C., 2018. Determination of epsilon for Omega vortex identification method. J. Hydrodyn. 30 (4), 541-548.

Ghahramani, E., Jahangir, S., Neuhauser, M., Bourgeois, S., Poelma, C., Bensow, R.E., 2020. Experimental and numerical study of cavitating flow around a surface mounted semi-circular cylinder. Int. J. Multiphas. Flow 124, 103191.

Green, S. (Ed.), 2012. Fluid Vortices, vol. 30. Springer Science \& Business Media. Harwood, C.M., Young, Y.L., Ceccio, S.L., 2016. Ventilated cavities on a surface-piercing hydrofoil at moderate Froude numbers: cavity formation, elimination and stability. J. Fluid Mech. 800, 5-56.

Hrubes, J.D., 2001. High-speed imaging of supercavitating underwater projectiles. Exp. Fluid 30 (1), 57-64. 
Huang, B., Zhao, Y., Wang, G., 2014. Large eddy simulation of turbulent vortexcavitation interactions in transient sheet/cloud cavitating flows. Comput. Fluids 92, 113-124.

Hunt, J.C., Wray, A.A., Moin, P., 1988. Eddies, Streams, and Convergence Zones in Turbulent Flows.

Jeong, J., Hussain, F., 1995. On the identification of a vortex. J. Fluid Mech. 285, 69-94.

Kinzel, M.P., Krane, M.H., Kirschner, I.N., Moeny, M.J., 2017. A numerical assessment of the interaction of a supercavitating flow with a gas jet. Ocean Eng. 136, 304-313.

Liu, C., Wang, Y., Yang, Y., Duan, Z., 2016. New omega vortex identification method. Science China Physics. Mech. Astron. 59 (8), 684711.

Liu, J.M., Wang, Y.Q., Gao, Y.S., Liu, C., 2019. Galilean invariance of Omega vortex identification method. J. Hydrodyn. 31 (2), 249-255.

Long, X., Cheng, H., Ji, B., Arndt, R.E., Peng, X., 2018. Large eddy simulation and Euler-Lagrangian coupling investigation of the transient cavitating turbulent flow around a twisted hydrofoil. Int. J. Multiphas. Flow 100, 41-56.

Michel, J.M., 1984. Some features of water flows with ventilated cavities. ASME J. Fluids Eng. 106 (3), 319-326.

Nicoud, F., Ducros, F., 1999. Subgrid-scale stress modelling based on the square of the velocity gradient tensor. Flow, Turbul. Combust. 62 (3), 183-200.

Pendar, M.R., Roohi, E., 2018. Cavitation characteristics around a sphere: an LES investigation. Int. J. Multiphas. Flow 98, 1-23.

Perry, A.E., Chong, M.S., 1987. A description of eddying motions and flow patterns using critical-point concepts. Annu. Rev. Fluid Mech. 19 (1), 125-155.
Robinson, S.K., 1990. A review of vortex structures and associated coherent motions in turbulent boundary layers. In: Structure of Turbulence and Drag Reduction. Springer, Berlin, Heidelberg, pp. 23-50.

Skidmore, G.M., Brungart, T.A., Lindau, J.W., Moeny, M.J., 2016. The control of ventilated supercavity pulsation and noise. Int. J. Multiphas. Flow 85, 14-22.

Tian, S., Gao, Y., Dong, X., Liu, C., 2018. Definitions of vortex vector and vortex. J. Fluid Mech. 849, 312-339.

Wang, C.C., Liu, Y., Chen, J., Zhang, F.Y., Huang, B., Wang, G.Y., 2019. Cavitation vortex dynamics of unsteady sheet/cloud cavitating flows with shock wave using different vortex identification methods. J. Hydrodyn. 31 (3), 475-494.

Wang, Y., Xu, C., Wu, X., Huang, C., Wu, X., 2017. Ventilated cloud cavitating flow around a blunt body close to the free surface. Phys. Rev. Fluids 2 (8), 084303.

Wang, Z., Huang, B., Zhang, M., Wang, G., Zhao, X., 2018. Experimental and numerical investigation of ventilated cavitating flow structures with special emphasis on vortex shedding dynamics. Int. J. Multiphas. Flow 79-95.

Wu, J.Z., Ma, H.Y., Zhou, M.D., 2007. Vorticity and Vortex Dynamics. Springer Science \& Business Media.

Wu, Q., Huang, B., Wang, G., Cao, S., 2018. The transient characteristics of cloud cavitating flow over a flexible hydrofoil. Int. J. Multiphas. Flow 99, 162-173.

Zhang, Y.N., Liu, K.H., Li, J.W., Xian, H.Z., Du, X.Z., 2018. Analysis of the vortices in the inner flow of reversible pump turbine with the new omega vortex identification method. J. Hydrodyn. 30 (3), 463-469.

Zhou, J., Adrian, R.J., Balachandar, S., Kendall, T.M., 1999. Mechanisms for generating coherent packets of hairpin vortices in channel flow. J. Fluid Mech. 387, 353-396. 